\title{
Pathobiology of Sjögren Syndrome: What do we Know?
}

\author{
Andres Pinto
}

Published online: 1 July 2014

(C) Springer International Publishing AG 2014

\begin{abstract}
Sjögren syndrome is a chronic autoimmune disorder that involves exocrine glands and multiple organs. This disorder presumably affects 1.3 million patients in the USA. The pathobiology of Sjögren syndrome has been studied for at least three decades, and reported mechanisms involve the activation of the innate immune system and the development of autoimmunity. The precise mechanisms and sequence of events leading to salivary and ocular dysfunction are still unclear, and apparently multiple pathways and risk factors condition an individual to the disease. This manuscript provides an overview of the current knowledge of the pathobiology underlying Sjögren syndrome.
\end{abstract}

Keywords Sjögren syndrome $\cdot$ Keratoconjunctivitis sicca . Xerostomia $\cdot$ Genetics $\cdot$ Dry mouth $\cdot$ Dry eye $\cdot$ T cells . B cells $\cdot$ Autoimmune $\cdot$ Pathology $\cdot$ Biology

\section{Introduction}

Sjögren syndrome (SS) is a chronic debilitating autoimmune disorder that occurs with multi-organ involvement. Frequent clinical characteristics of SS are ocular and oral involvement, defined by sicca symptoms. Xerophthalmia and xerostomia accompany other symptoms of SS and may involve neurologic, pulmonary, and rheumatologic manifestations [1-3, 4•]. Originally thought to affect only exocrine glands, SS is now known to have widespread effects, and its expression can overlap with other connective tissue and immune-mediated disorders.

\footnotetext{
A. Pinto $(\square)$

Oral and Maxillofacial Medicine and Diagnostic Sciences,

University Hospitals Case Medical Center and Case Western Reserve

School of Dental Medicine, 2124 Cornell Rd., Rm 1190, Cleveland, OH 44106, USA

e-mail: Andres.pinto@UHhospitals.org
}

Oral health providers are an integral part of the multidisciplinary approach to the diagnosis and management of SS $[3,5-7]$. They contribute to the diagnosis of SS, the longterm management of salivary dysfunction/hypofunction, and the hard and soft tissue sequelae of SS [7]. An oral environment devoid of moisture poses a dramatic challenge to patients and providers alike, and long-term proactive management of dry mouth is recommended for patients suffering from SS.

\section{Epidemiology}

The prevalence of SS is elusive, in part due to the existence of multiple diagnostic and classification algorithms. The changing nature of these approaches makes estimation of epidemiologic figures very difficult. It seems that SS afflicts mostly women ( $9: 1$ female to male ratio) in the fourth to fifth decades of life [5]. In the USA, it is estimated that SS affects at least 1.3 million individuals, and that many more are symptomatic but undiagnosed [8]. Symptom prevalence figures in the USA report approximately 5 million individuals suffering from dry eye symptoms, and the prevalence of xerostomia has been calculated to be as high as 75 million [9]. European studies describe the prevalence of SS as being between 0.1 and $0.4 \%$ of the population in areas of the UK and Greece [10]. Comorbid presence of autoimmune disease and relatives with autoimmune disorders are significant risk factors for development of SS.

\section{Diagnosis}

The diagnosis of SS has been the focus of extensive research, with two major classification strategies emerging in the last 12 years [11]. With the establishment of these consensus statements, there appears to be more consistency going forward regarding the diagnosis of this disorder $[9,11,12]$. 
Classification schemes for SS have evolved from a combination of subjective and objective findings (serology and ocular staining being part of the later), to mostly objective strategies described in the recent American College of Rheumatology guidelines $[11,13]$. This progression reflects a deeper understanding of the pathobiology underlying the development of SS, which has significantly advanced in the last two decades. In general, SS can be divided into primary or secondary, depending on the presence of co-morbid rheumatologic or autoimmune disease. Elucidation of the pathobiology of SS is important not only to guide treatment, but also to refine diagnostic algorithms for the disorder. The objective of this article is to offer a brief overview of the current understanding of the pathobiology of SS, with focus on salivary manifestations (Table 1).

\section{Salivary Manifestations}

Immune changes in salivary glands were identified in the 1970s by Greenspan, Daniels, and others, and led to the current histologic classification regarding salivary gland involvement [12]. Lymphocytic infiltration of exocrine tissues is described as predominantly of T cell or B cell lineage. Studies have confirmed the stable nature of lymphocytic infiltration of tissues during the course of the disease, with the exception of patients who have prognostic factors for the development of lymphoma $[12,14 \bullet, 15 \bullet \cdot]$. Glandular T cell infiltration leads to a persistent inflammation that results in the clinical characteristics of the disorder. Extensive histologic evaluation of minor salivary glands in SS reveals areas of ectopic germinal lymphatic centers, perhaps associated with the risk of developing lymphoma [10]. Inflammation plays a significant role in this local process at many levels, including secretion of

Table 1 Select advances in the understanding of the pathobiology of Sjögren syndrome

\begin{tabular}{ll}
\hline Mechanism & Examples \\
\hline Triggers of innate immunity & $\begin{array}{c}\text { Retrovirus, infection, age?, gender?, } \\
\text { genetics?, hormonal? }\end{array}$ \\
T cell innate immune response & $\begin{array}{c}\text { TH1, TH2, TH17 pathways, role } \\
\text { of SGECs }\end{array}$ \\
$\begin{array}{l}\text { Adaptive autoimmune } \\
\text { response }\end{array}$ & B cell activation, IFN, TNF \\
$\begin{array}{l}\text { Inflammation } \\
\text { Autoantibodies }\end{array}$ & $\begin{array}{l}\text { Interleukin secretion and proliferation } \\
\text { anti-Ro(SSA), anti-La(SSB), ANA, } \\
\text { rheumatoid factor }\end{array}$ \\
Epigenetics & Demethylation, miRNA, retroviral \\
& effects
\end{tabular}

ANA anti-nuclear antibodies, IFN interferon, miRNA microRNA, SGECS salivary gland epithelial cells, $T H$ T helper, $T N F$ tumor necrosis factor autoantibodies and potent cytokines and interferon (IFN). The exact mechanism by which this infiltrate replaces salivary tissues is yet unknown. Salivary gland epithelium cells (SGECs) are capable of recruiting inflammatory mediators and virtually any immune cell, enhancing the process of inflammation and progressive apoptosis [12, 14•]. A complex interaction exists between SGECs and T cells, and this mechanism is amplified in SS patients' salivary tissues. In fact, SGECs are believed to play a crucial role in the development of anti-Ro and anti-La antibodies and potentiation of their immune response [14•]. Potential triggers for this response are not clear, and may include viral or other infections [16].

Additional immune cells are also found in the inflammatory infiltrate and include macrophages, natural killer (NK) cells, and dendritic cells. Dysfunctional performance of dendritic cells is observed in salivary SS, and theories supporting this change are based on altered antigen-presenting and selftolerance mechanisms. [10] $\mathrm{T}$ helper (TH)1 responses are associated with mild lesions, whereas TH17 responses are linked to more advanced stages of the disease. Preliminary evidence exists on the role of retroviral infections causing SSlike symptoms in healthy and immune-suppressed patients. Retroviral activity has been detected in affected SS salivary gland tissue of patients who are infected with human immunodeficiency virus (HIV) and may develop SS clinical manifestations (i.e., parotid infiltration). These clinical changes are reversed with the use of antiretroviral therapy [16].

\section{Autoantibodies}

Among several autoantibodies described in SS, anti-Ro(SSA), anti-La(SSB), ribonucleic proteins, anti-fodrin, anti-nuclear antibodies (ANA), rheumatoid factor, and anti-M3R (muscarinic acetylcholine receptors), are worth noting $[17,18]$.

Subtypes of anti-Ro antibodies (anti-Ro52 and anti-Ro60) are significant due to their association to disease subtypes and progression. For instance, anti-Ro52 has $66.7 \%$ specificity, while anti-Ro60 has $52.1 \%$ specificity to SS. The presence of these markers is linked to longer disease duration prior to diagnosis, recurrent parotid gland enlargement, and severity of minor salivary gland infiltration $[14 \bullet, 15 \cdot \bullet]$. The appearance of rheumatoid factor is common, and has been reported to have a prevalence as high as $74 \%$ (with a lower range of $36 \%$ ) and to be linked to a younger age at diagnosis and multi-organ involvement [13, 14•]. Additional autoantibodies such as anti-smooth muscle antibodies (ASMA), anti-smith antibodies (AMA), cryoglobulins, and anti-cyclic citrullinated antibodies (anti-CCP) are present in less than $20 \%$ of patients $[12,19,20]$. Patterns of antibody presentation have important disease implications: cryoglobulins are indicators of severe disease and risk of lymphoma, anti-CCP, although infrequent, may indicate the presence of none-erosive arthritis, and anti- 
centromere antibodies are linked to overlapping phenotypes of SS and scleroderma $[6,19,20]$. These tests are readily available to the clinician for evaluation of a suspected SS patient.

\section{Ocular Manifestations}

Keratoconjunctivitis sicca, or dry eye, is a common complaint seen in SS patients and affects quality of life. Patients complain of "sandy" sensations in the eyes and itching. Several mechanisms have been proposed to describe the ocular involvement in this disorder. Among these, infection (viral), moebian gland dysfunction, and hormonal imbalance are noteworthy [8].

Apoptosis plays a relevant role in the development of ocular complications. A major process associated with ocular (and salivary) involvement is the cleavage of alpha-fodrin, a protein involved in membrane composition and function. Its breakdown by caspase 3 produces fragments that may act as tissue-specific antigens. Antibodies against alpha-fodrin have been found in $64 \%$ of patients with SS [1, 8, 21].

\section{Neuronal Effects}

Exocrine glands are heavily innervated by parasympathetic and sympathetic fibers. Deficiency in tear and saliva secretion may be related to the effect of cytokines that interfere with neural transmission [2]. Antibodies against M3R have the effect of blocking neurogenic reception to acinar cells, either by causing internalization of the receptors or by causing progressive desensitization of these receptors. The constant receptor binding may cause significant reduction in the secretory capacity of glandular acini $[8,18]$.

\section{The Role of T Cells}

The proposed immune-mediated mechanisms are initiated by delayed glandular genesis and increased apoptosis $[13,22 \bullet \bullet$, 23]. These events may be followed by leukocyte migration with the recruitment of $\mathrm{T}$ and eventually B lymphocytes. One of these pathways may be the activation of dendritic cells and production of IFN- $\alpha$ by one of many mechanisms previously discussed. This causes sustained further apoptosis and necrosis of glandular tissue, and upregulation of major histocompatibility complex (MHC) class I and II molecules [23]. Prolonged immune activation is facilitated by IFN- $\gamma$, and interleukin (IL)-6, -10, and -15, leading to impairment of secretory function secondary to the development of an autoimmune response $[22 \bullet \cdot]$. In addition, M3R-activated T cells may induce early events leading to salivary sialadenitis. Activated T cells, via IFN- $\alpha$ and IL-17, prolong the inflammatory presence [24]. A close association exists with several B cell epitopes and the prevalence of $40-50 \%$ of antiM3R antibodies in serum of SS patients, making them potential candidates for further analysis and targeted treatment $[14 \bullet$, 17]. Of note, $\mathrm{M} 3$ receptors are ubiquitous of exocrine glands, particularly salivary, and are targeted by existent pharmacologic approaches to increase salivary flow [25]. Another change in the metabolic activity of T cells in SS is at the level of T cell receptors (TCR). Conformational changes in TCRs are responsible for $\mathrm{T}$ cell subtypes found in glandular and ocular tissues, and may be another option for future therapeutic options.

This rather simplistic model still leaves many questions unanswered and does not necessarily imply a linear progression of SS. It has become more apparent that maturity of the disease is linked to an intense inflammatory response that is followed by an adaptive immune reaction [24]. T cell activity in SS can be broadly defined as occurring via TH1, TH2, and TH17 pathways.

\section{T Helper (TH)1 and TH2 Pathways}

These TH cells are responsible for initiation of cell-mediated immunity. Among other cells, $\mathrm{CD} 8^{+} \mathrm{T}$ cells, macrophages, and NK cells are recruited. Secretion of IL-4, IL-5, and IL-15 assist the enrollment of B cells and activation of humoral immunity. Secretion of IFN- $\gamma$ leads to delayed glandular formation, and activation of adhesion molecules including vascular cell adhesion molecules (VCAM) and intracellular cell adhesion molecules (ICAM). These promote further migration of inflammatory cells into the tissues and expansion of the apoptotic and autoimmune effects $[22 \bullet \cdot, 26]$. This movement is set in motion by the production of chemotactic factors or chemokines produced by $\mathrm{T}$ cells [27].

\section{TH17 Pathways}

TH17 cells have recently been described. Their action is independent of both $\mathrm{TH} 1$ and $\mathrm{TH} 2$ pathways. These cells secrete IL-17 and IL-17f in response to stimulation by IL-6 and transforming growth factor (TGF)- $\beta$. The effect of the TH17 response is the intense prolongation of the inflammatory response and the further production of several interleukins, which maintain the TH17 activity. Potentiation of metalloproteinase (MMP) activity has a particularly effective role on these pathways, and MMPs are responsible for persistent tissue damage.

An important dysregulation of tumor necrosis factor (TNF)- $\alpha$ receptors has been reported in SS patient serum and salivary tissues. This mechanism controls inflammation and is fundamental in the genesis of B cell-activating factor (BAFF). Over-expression of BAFF is reported in SS patients 
and reflects the loss of self-tolerance and antibody production, described in patients who develop lymphoma $[25,26]$.

\section{The Role of B Cells}

B cells have gained prominence for their role in the pathologic process of SS. B cells in the early stage of development (transitional B cells) were detected in salivary tissues of SS patients, supporting the early development of autoantigens in these tissues [28• 29]. Further, the clonal expansion of B cell lineage argues against a minor role of B cells in the pathogenesis of SS. Although the actions described above by $\mathrm{T}$ cells assume a direct association between $\mathrm{T}$ cell activation and $\mathrm{B}$ cell activation, B cells are also responsible for promoting additional immune response via antibody-dependent and independent mechanisms. [30] There is evidence that B cells may be responsible for the growth of germinal centers surrounding minor salivary gland histology. These cells may also act as antigen-presenting cells to $T$ cells. Increased levels of BAFF reported in salivary gland tissue, serum, and saliva of SS patients provide additional support to this theory [29].

\section{Epigenetics}

Epigenetics is defined as modified gene expression without a change of DNA sequence. Mechanisms of this type control organ development and sequence of genetic expression with environmental variations [31]. Described changes in SS include induced hypomethylation, human endogenous retroviruses, DNA demethylation, modification of histones, and deregulation of microRNA (miRNA).

\section{Induced Hypomethylation}

The evidence supporting hypomethylation changes derives from the use of medications to control hypertension in animal models, causing SS symptoms. An example among these drugs is hydralazine.

\section{Human Endogenous Retroviruses}

The human endogenous retroviruses are ubiquitous and usually are not pathogenic. Several studies have identified reactivity between SS antibodies and several retroviral antigens, thus posing the interesting question about the possible role of retroviral etiology in SS. Polymerase chain reaction assessment of minor salivary tissue of SS patients detected retroviral elements with the corresponding presence of autoantibodies towards them [31]. Therefore, the potential implication of these viruses in the pathogenesis of SS remains an important venue for future research.

DNA Demethylation and Histone Modification

Multiple in vivo and in vitro studies demonstrate increased DNA demethylation in patients with SS when compared with controls $[31,32 \bullet \cdot]$. These changes have been reported in SGECs of SS patients in vivo and in culture, and are related to disease activity and intensity of B cell infiltration. Data from patients treated with anti-B cell therapy evidence increased methylation compared with controls, underscoring the role of B cells in promoting DNA demethylation [32•*]. Histone modification may follow DNA demethylation and is reported in autoimmune disorders besides SS, such as systemic lupus.

microRNA

Micro RNAs are very small segments of RNA produced after transcription. They bind to segments of messenger RNA causing degradation and dysregulation of RNA activity. These molecules are associated to the development of inflammatory autoimmune disorders [32••]. In SS, miRNA activity seems to affect several mechanisms in tandem. The presence of miRNA in salivary gland tissue affects neurologic secretory control and the expression of autoantigens Ro and La. Moreover, elevated levels of miRNA are identified in peripheral blood cells of SS patients prior to the occurrence of symptomatic disease. Specific miRNA such as MiR146a and MiR146b have wide effects on proapoptotic pathways and sensitization of toll-like receptors and IL-1 receptors $[32 \bullet \bullet$.

\section{Conclusion}

Major progress has been achieved in the past 30 years in the understanding of the pathobiology of SS. The initial description of inflammatory periductal infiltration in salivary glands opened the door for many questions (i.e., components of the infiltration, type of cellular responses, autoantibodies), some of which have been answered by murine, human, and in vitro studies. Clearly, secretory pathways in salivary glands and ocular glands are initially affected by an innate immune response that progressively transforms into a sustained adaptive immune (autoimmune) activity. The role of SGECs in this process is salient, as they interact with the immune system in the development and antigen presentation at local and possibly systemic levels. What remains to be discovered is the precise mechanism by which the initial immune response is set in motion, and which patient-related and environmental 
factors are early predictors of the disease (i.e., stress, hormonal, infections, environmental). Current understanding of SS encompasses multiple pathways and mechanisms that cover immune dysfunction, prolonged inflammatory-mediated tissue necrosis, loss of neurologic glandular control, and changes at the epigenetic levels, all sharing common themes. These commonalities are the apoptotic process in exocrine tissue, generation of self-antigens and loss of tolerance, and the preponderance of lymphocytic infiltration in the affected glands. To make matters more interesting, much of the current research has been performed in patients with primary SS, and less is known about the pathobiology and physiology of secondary SS.

The implications of these findings to the clinician highlight the timely identification of symptoms of SS and the ability to understand its clinical presentation. SS remains a vastly unrecognized disease, and oral health providers are major players in the aggressive management of the oral effects of SS. Fundamental questions that demand answers include the identification of predictive intrinsic (patient centered) and extrinsic factors for development of the disease, elucidation of key elements that trigger the innate immune response, and the limitation or delay of exocrine gland necrosis. These very ambitious questions set the stage for future research in SS that will lead to improvements in diagnosis and groundbreaking therapeutics.

\section{Compliance with Ethics Guidelines}

Conflict of Interest Dr. Andres Pinto is a section editor for Current Oral Health Reports.

Human and Animal Rights and Informed Consent This article does not contain any studies with human or animal subjects performed by any of the authors.

\section{References}

Papers of particular interest, published recently, have been highlighted as:

- Of importance

•. Of major importance

1. Ng WF, Bowman SJ. Primary Sjogren's syndrome: too dry and too tired. Rheumatology (Oxford). 2010;49(5):844-53.

2. Chai J, Logigian EL. Neurological manifestations of primary Sjogren's syndrome. Curr Opin Neurol. 2010;23(5):509-13.

3. Mathews SA, Kurien BT, Scofield RH. Oral manifestations of Sjogren's syndrome. J Dent Res. 2008;87(4):308-18.

4. Pavlakis PP, Alexopoulos H, Kosmidis ML, et al. Peripheral neuropathies in Sjogren's syndrome: a critical update on clinical features and pathogenetic mechanisms. J Autoimmun. 2012;39(1-2): 27-33. Thorough review of neurologic sequaelae of SS.

5. Niessen LC, Gibson G, Kinnunen TH. Women's oral health: why sex and gender matter. Dent Clin North Am. 2013;57(2):181-94.
6. Carr AJ, Ng WF, Figueiredo F, et al. Sjogren's syndrome - an update for dental practitioners. Br Dent J. 2012;213(7):353-7.

7. Wu AJ. Optimizing dry mouth treatment for individuals with Sjogren's syndrome. Rheum Dis Clin North Am. 2008;34(4): 1001-10.

8. Nguyen CQ, Peck AB. Unraveling the pathophysiology of Sjogren syndrome-associated dry eye disease. Ocul Surf. 2009;7(1):11-27.

9. Sankar V, Noll JL, Brennan MT. Diagnosis of Sjogren's syndrome: American-European and the American College of Rheumatology classification criteria. Oral Maxillofac Surg Clin North Am. 2014;26(1):13-22.

10. Jonsson R, Vogelsang P, Volchenkov R, et al. The complexity of Sjogren's syndrome: novel aspects on pathogenesis. Immunol Lett. 2011;141(1):1-9.

11. Rasmussen A, Ice JA, Li H, et al. Comparison of the AmericanEuropean Consensus Group Sjogren's syndrome classification criteria to newly proposed American College of Rheumatology criteria in a large, carefully characterised sicca cohort. Ann Rheum Dis. 2014;73(1):31-8.

12. Moutsopoulos HM. Sjogren's syndrome: a forty-year scientific journey. J Autoimmun. 2014;51C:1-9.

13. Vissink A, Bootsma H, Spijkervet FK, et al. Current and future challenges in primary Sjogren's syndrome. Curr Pharm Biotechnol. 2012;13(10):2026-45.

14. Kyriakidis NC, Kapsogeorgou EK, Tzioufas AG. A comprehensive review of autoantibodies in primary Sjogren's syndrome: clinical phenotypes and regulatory mechanisms. J Autoimmun. 2014;51C: 67-74. Important review of autoantibodies in SS.

15.・ Nocturne G, Mariette X. Advances in understanding the pathogenesis of primary Sjogren's syndrome. Nat Rev Rheumatol. 2013;9(9):544-56. This manuscript describes in detail advances in the understanding of the biologic process underlying SS.

16. Sipsas NV, Gamaletsou MN, Moutsopoulos HM. Is Sjogren's syndrome a retroviral disease? Arthritis Res Ther. 2011;13(2):212.

17. Sumida T, Tsuboi H, Iizuka M, et al. The role of M3 muscarinic acetylcholine receptor reactive T cells in Sjogren's syndrome: a critical review. J Autoimmun. 2014;51C:44-50.

18. Ferraccioli G, De Santis M, Peluso G, et al. Proteomic approaches to Sjogren's syndrome: a clue to interpret the pathophysiology and organ involvement of the disease. Autoimmun Rev. 2010;9(9):622-6.

19. Ramos-Casals M, Stone JH, Cid MC, Bosch X. The cryoglobulinaemias. Lancet. 2012;379(9813):348-60.

20. Bournia VK, Vlachoyiannopoulos PG. Subgroups of Sjogren syndrome patients according to serological profiles. J Autoimmun. 2012;39(1-2):15-26.

21. Voulgarelis M, Tzioufas AG. Pathogenetic mechanisms in the initiation and perpetuation of Sjogren's syndrome. Nat Rev Rheumatol. 2010;6(9):529-37.

22.• Karabiyik A, Peck AB, Nguyen CQ. The important role of T cells and receptor expression in Sjogren's syndrome. Scand J Immunol. 2013;78(2):157-66. Excellent review on the role of T cells in SS initiation and progression.

23. Katsifis GE, Moutsopoulos NM, Wahl SM. T lymphocytes in Sjogren's syndrome: contributors to and regulators of pathophysiology. Clin Rev Allergy Immunol. 2007;32(3):252-64.

24. Li H, Ice JA, Lessard CJ, Sivils KL. Interferons in Sjogren's syndrome: genes, mechanisms, and effects. Front Immunol. 2013;4:290.

25. Kallenberg CG, Vissink A, Kroese FG, Abdulahad WH, Bootsma $\mathrm{H}$. What have we learned from clinical trials in primary Sjogren's syndrome about pathogenesis? Arthritis Res Ther. 2011;13(1):205.

26. Lisi S, Sisto M, D'Amore M, Lofrumento DD, Ribatti D. Emerging avenues linking inflammation, angiogenesis and Sjogren's syndrome. Cytokine. 2013;61(3):693-703. 
27. Roescher N, Tak PP, Illei GG. Cytokines in Sjogren's syndrome. Oral Dis. 2009;15(8):519-26.

28. Cornec D, Devauchelle-Pensec V, Tobon GJ, et al. B cells in Sjogren's syndrome: from pathophysiology to diagnosis and treatment. J Autoimmun. 2012;39(3):161-7. Good review on the role of B cells.

29. Youinou P, Devauchelle V, Hutin P, et al. A conspicuous role for B cells In Sjogren's syndrome. Clin Rev Allergy Immunol. 2007;32(3):231-7.
30. Youinou P, Devauchelle-Pensec V, Pers JO. Significance of B cells and B cell clonality in Sjogren's syndrome. Arthritis Rheum. 2010;62(9):2605-10.

31. Le Dantec C, Varin MM, Brooks WH, et al. Epigenetics and Sjogren's syndrome. Curr Pharm Biotechnol. 2012;13(10):2046-53.

$32 . \bullet$ Konsta OD, Thabet Y, Le Dantec C, et al. The contribution of epigenetics in Sjogren's Syndrome. Front Genet. 2014;5:71. Discussion of current epigenetic evidence supporting SS. 